\title{
Large-Scale Structure of the Dynamic Steady State Universe
}

\author{
Conrad Ranzan \\ Cosmology Dept., DSSU Research, Niagara Falls, Ontario, Canada
}

\section{Email address:}

ranzan@cellularuniverse.org

\section{To cite this article:}

Conrad Ranzan. Large-Scale Structure of the Dynamic Steady State Universe. American Journal of Astronomy and Astrophysics. Vol. 4, No. 6, 2016, pp. 65-77. doi: 10.11648/j.ajaa.20160406.11

Received: May 17, 2016; Accepted: October 13, 2016; Published: November 1, 2016

\begin{abstract}
The conventional view is that the cosmic spectral-redshift, an effect observed in all directions of the heavens, is primarily a measure of space-medium expansion. Significantly, the more distant the galaxy the greater is the redshift —and the greater is the intervening space expansion. The redshift evidence is unequivocal; its implication, its interpretation, however, is not. The important question is this: Does all the evidence for "space" expansion necessarily imply that the whole visible universe must also be expanding? Given a universe whose volume consists mostly of expanding space, does it follow that the entire universe must expand? On this momentous issue rests the future course of cosmology. Yet, it seems, the question has never been seriously addressed. Meanwhile, there is a growing awareness that the assertion of whole-universe expansion is a non-sequitur fallacy. Universe expansion is the unscientific extrapolation of space expansion. It is an unwarranted extrapolation of general relativity. Instead of universal expansion, this paper considers regional space expansion along with regional space contraction - one dynamic process being the complement of the other. This dual dynamic nature of the medium is given full recognition and exploited in the construction of a nonexpanding cellular model of the Universe. The emphasis is on the cell structure and the two "space" postulates involved in sustaining the structure, for it is in the structure of the cosmic cells that the nature of the relationship between Lambda and gravity is revealed.
\end{abstract}

Keywords: Cosmology, Cosmic Redshift, Cellular Structure, Cosmic Cells, Galaxy Structures, Dynamic Steady State Universe, Lambda, Gravity, Space Expansion, Space Contraction

\section{Introduction}

While the question of a reason for the repulsion issuing from the positive energy of empty space doesn't lead us anywhere, it makes sense to ask how this repulsion will manifest itself. -Henning Genz [1].

Based on the fact that there is simply no way to determine from the redshift alone whether space is expanding in an expanding universe, or expanding within a non-expanding universe this paper explores how "this repulsion will manifest itself" - how the space expansion will manifest itself on the cosmic scale. The key factor in countering expansion will be examined.

Rather than attempt to confine the cosmic space expansion, the traditional approach has been to extrapolate it into the expansion of the whole universe. General relativity, a localized theory of gravity has been extrapolated into the highly speculative Big Bang hypothesis! One of the world's most famous astronomers (and unquestionably the world's expert on peculiar galaxies), Halton Arp (1927-2013), has collected the evidence over many years and maintains that extragalactic redshifts are not caused by an expanding universe [2]. His accumulated evidence is incompatible with an interpretation of universal expansion, and undermines the very foundation of all big bang (BB) models. If there is no universe-wide expansion, then the notion of a big explosion falls apart - there was no big bang.

Halton Arp recognized the crisis in his field, knows it is on the wrong path, and struggles, in his words, "to get the mainstream of astronomy back on track."

"I believe the observational evidence has become overwhelming, and the Big Bang has in reality been toppled. There is now a need to communicate the new observations, the connections between objects and the new insights into the workings of the universe - all the primary obligations of academic science, which has generally tried to suppress 
or ignore such dissident information." [3].

The physicist/cosmologist Hannes Alfvén (1908-1995), a Nobel Laureate and specialist in plasma physics, was another who came to recognize the fanciful and implausible nature of universal expansion. He saw what its backward-in-time extrapolation implied and rejected it outright. The universe is not something that issued from some genesis event; rather, the universe is something that simply is. To him it was patently evident that the big bang never happened!

"There is no rational reason to doubt that the universe has existed indefinitely, for an infinite time. It is only myth that attempts to say how the universe came into being either four thousand years ago or twenty billion years ago." [4].

\section{Distinguishing Between What Expands and What Does Not Expand}

The real universe does not expand. There is a generally accepted axiom of cosmology known as the cosmological principle. It states that the universe is homogeneous (constant density) and isotropic (the same in all directions). In practical terms it means there can be no preferred location. It means a universe can have no boundary that separates itself from some outer region that is not a part of itself. And it can have no center point.

Now given that the BB is an expanding universe, supposedly having grown from the size of a primordial atom to become, some 13.7 billion years later, its present size, and since it has a measurable (and expanding) diameter, one might argue that it has a limiting boundary. We might think of it as a cosmic edge dividing such an expanding universe from an outer region of nothingness (or whatever) that it is expanding into; and then conclude that the cosmological principle was being violated. This argument would be devastating to a simple expanding-universe model. But if applied against the BB model, the argument would be considered conceptually unsophisticated. The reason for its inapplicability rests with the fact that the BB is merely a mathematical construction -albeit of extraordinary sophistication. Equations based on Einstein's general relativity can be used to show that the BB universe expands while complying with the cosmological principle (or at least some weaker version of it). For example, the forbidden boundary-edge can be made to vanish by formulating a curved cosmic space into a four-dimensional sphere - a mathematical hypersphere. This makes the BB universe "finite yet unbounded."

The dreaded cosmic edge can be cleaned up by just wrapping it around a mathematical four-dimensional construction. Then, when an object or a light beam reaches the "boundary," it theoretically re-emerges from the diametrically opposite boundary. Mathematically, it is even possible to have a universe that somehow expands into itself.

But the real world is not a mathematical construction - not a Platonic metaphysical world. In the real world, the question remains, what does the expanding universe expand into?

Cosmology demands a strong interpretation of the cosmological principle; otherwise the universe would be unintelligible [5]. Nevertheless, the BB model restricts itself to a weak version of the principle. In the weak version, the universe varies with time.

The BB starts out small, really small, and at some later time it is bigger! The BB exists in a state of great density and at some later time it has transformed into a voids-dominated patchwork of clustered matter! The BB starts out hot, really hot; at a later time it is cold; and is predicted to be really cold in some future time. Without question, the BB model - by demanding whole-universe expansion - violates the axiom necessary for a cogent cosmology.

Predictably, the universe, based on the BB hypothesis, is made unintelligible. Astronomer Neil deGrasse Tyson calls it The Inexplicable Universe. American astrophysicist S. M. Carroll forthrightly calls it "the Preposterous Universe," "and admits that "the universe we observe seems dramatically unnatural" and, in fact, it "staggers under the burden of its unnaturalness."

The real World is a natural world. It is the very manifestation of Existence. Existence, we recognize, is absolute - it does not permit of modification or of restrictions or of conditionals. Existence has no spatial or temporal limits; existence is infinite. And so, the natural Cosmos has no cosmic edge, no preferred location, no preferred time, no initial conditions, no time-dependent states.

If one's theory of the Universe is to comply with a strong cosmological principle then the universe it purports to describe must surely be infinite in extent; and to discuss the expansion of an infinite universe is rather pointless. A true infinite universe is already fully expanded. One is forced to make a choice: either reject the strong cosmological principle or accept the reasonable and probable notion that our Universe does not expand.

But what about the evidence? What about the irrefutable evidence that all distant galaxies, in all directions of the heavens, display a cosmic redshift? Moreover, the degree of redshift is nearly in direct proportion to the source galaxy's distance. And given that this redshift (RS) is caused by the expansion of space (as almost all cosmologists will agree) then it would seem there can be only one interpretation - galaxies, in every direction, are all receding and the greater the RS-distance the faster they are racing away. Apparently, the universe is exploding. The evidence seems solid enough. Indeed, it was deemed solid enough to use as one of the four pillars of BB cosmology.

However, consider the words of the man who is credited with discovering the expansion of space (or more properly the expansion of the space medium):

“... if redshift are not primarily due to velocity shift ... the velocity-distance relation is linear, the distribution of the nebula is uniform, there is no evidence of expansion, no trace of curvature, no restriction of the time scale ... and we

1 S. M. Carroll even used the term for the name of his website http://PreposterousUniverse.blogspot.com 
find ourselves in the presence of one of the principles of nature that is still unknown to us today ... whereas, if redshifts are velocity shifts which measure the rate of expansion, the expanding models are definitely inconsistent with the observations that have been made ... expanding models are a forced interpretation of the observational results" -Edwin Hubble [6].

In other words, Hubble did not accept the notion that distant galaxies were receding as part of a whole-universe expansion; in fact, he did not believe that distant galaxies were receding at all.

There is no question that the redshift is related to distance. It is well understood that the expansion of intervening space induces a RS. But what is not being recognized is that regions of contracting space are also involved in causing the light to become redshifted as has been shown in [7] and [8]. Consequently, it does not follow that the Universe is expanding. It is simply not necessary to blow-up the entire universe to explain the cosmic RS.

There is no question that the redshift is related to distance. It is today well understood that the redshift is caused primarily by the expansion of intervening space. However, it does not follow that the Universe is expanding. It is simply not necessary to blow-up the entire universe to explain the cosmic RS.

So, what is expanding? If the Universe is not expanding, then what is?... Of course, space is expanding. But we already know that. The question should be, what is this space that is expanding? The conventional wisdom maintains that space (Einstein's spacetime) is not absolute and can only be represented spatially by 4-dimensional geometry. However, the space of the Dynamic Steady State Universe (DSSU) is completely permeated by a medium. It may, therefore, be said that DSSU space has a certain absoluteness quality (but unlike Newtonian space, which was absolute but passive and immovable, DSSU space is measurable and active). Both types of space, Einstein's spacetime and DSSU's space medium, are dynamic and both expand.

For Einstein's non-absolute space, the dynamic process can be described as a mysterious geometric expansion - $\mathrm{a}$ mathematical stretching of coordinate intervals.

For DSSU's quasi-absolute space medium, the dynamic process is a growth, or addition, of new aether-like space.

The fact that this redshift effect is observed provides the observable "proof" that the space medium, the carrier of electromagnetic waves, does expand. When light waves travel through expanding space over great distances they become elongated. For instance, the yellow light from a typical star will be stretched towards some longer wavelength (in the direction of the red-color part of the light spectrum).

There is also a theoretical argument to support "space" expansion.

According to Einstein's general relativity, space must be dynamic, it simply cannot remain static. Using the Einstein equations, the Dutch astronomer Willem de Sitter, in 1917, made the discovered that a region of space - in which there was no matter- must expand. What this means is that space itself must expand (in the absence or near absence of mass). De Sitter called his cosmological model "solution B" in deference to Einstein's original "solution A." Science historian Corey Powell explains in [9]:

While analyzing Einstein's equations, de Sitter found that they could be reformulated in ways that Einstein had not considered. In a trio of dense papers presented to the Royal Astronomical Society in 1917, de Sitter set out three slightly different interpretations of this discovery. In the final one, he hit on a strange but useful simplification and set the density equal to zero. This "de Sitter universe" maintained key elements of Einstein's [1917 static] universe -it was still uniform and it still had a tacked-on Lambda- but it contained no matter. He called the... model "solution B"...

Essentially, deSitter removed the effect of space curvature from the equations and allowed space to be "flat"; then he gave 4 , Einstein's famous cosmological constant, a positive value. The result: Lambda became the "force" that expands space.

It should be pointed out that the dynamic quality of space has a dual aspect: the equations permit both expansion and contraction. More on this dual nature later. Significantly, the deSitter effect applies irrespective of the absolute or non-absolute nature of space. If we could give Einstein's mathematical (geometrodynamical) space a physical reality it will still expand. If we define space as an ethereal medium then it too will expand.

The property of space expansion was affirmed by Alexander Friedmann when he published two noteworthy papers in the journal Zeitschrift für Physik. "On the Curvature of Space" appeared in 1922; "On the Possibility of a World with Constant Negative Curvature" appeared in 1924. Friedmann used the equations of general relativity, to which he had applied a correction, but instead of using Lambda as the parameter for space expansion as deSitter had done years earlier, Friedmann set Lambda equal to zero and made curvature the parameter for space expansion. He used the purely mathematical concept of curvature (a 4-dimentional geometric curvature) and compliantly denied the absoluteness of space. Absolute or non-absolute, once again it was shown that space could not be static; it had to expand (or contract). [10].

Space expansion is based on a proven theory. Although - as an unfortunate consequence of the wrong conclusion extracted from the Michelson-Morley experiments - Einstein meant it as a pure geometric interpretation, there are valid reasons for giving it a physical dynamic interpretation. And that is what DSSU theory does, it defines space as an ethereal medium having two measurable properties - the ability to dynamically expand and contract.

\section{Where Does All The Expanded Space Go?}

"According to the doctrine of the harmony of opposites, a process demands that there be a harmonious opposing process. There must be a process to counteract the 
established process of space expansion. The harmony of opposites requires our universe to have a balancing space contraction." [11].

Having established that space expands but the Universe does not, an obvious question arises. Where does all this continually expanding space end up?

Let us for a moment go back to "solution B" - deSitters vacuous region of $\Lambda$-induced expanding space. Einstein had intended that $\Lambda$ should stabilize the universe and make it more or less static; and had assumed that $\Lambda$ would guarantee there could be no solution for the case in which the cosmic density is zero. He strongly objected to the run-away expansion implied by the deSitter solution.

As it is detailed in C. S. Powell's book, God in the Equation: In a letter to de Sitter, Einstein complained that the empty universe "does not correspond to any physical possibility." But now he clearly knew he had not attained the one and only description of reality, what he called "the true state of affairs." The prophecy of 1917 [of a static universe] remained to be fulfilled. [12].

Now, note carefully what Einstein was rejecting. An empty universe "does not correspond to any physical possibility."... Yes, but what about an empty region of the Universe? (At this pivotal moment History missed the opportunity of initiating a revolution in cosmology.) What about the Voids, those vast regions virtually empty of ponderable material?!

Unfortunately, in those early years of the $20^{\text {th }}$ century, voids of such a scale were not evident and not even imagined. It is fascinating to speculate what might have been. If Einstein had known of the existence of Voids 300 million lightyear across, would he have made the connection with the deSitter solution? Would he have recognized them as solution $B$ regions?

What Einstein effectively rejected was that the Universe is filled with deSitter-effect regions —with each region expanding its own space, its own medium!

Returning to the question of how to curb space expansion: The answer is right there in front of us. General relativity says that space is dynamic, and both dynamic expansion and dynamic contraction are permitted. The answer is that expansion is curbed by space contraction. Expansion in one region is countered by contraction in another region. The same set of equations but with different parameter values.

And the evidence for space contraction is unequivocal. For example, the space contraction occurring within the Earth holds you in your chair, keeps your coffee in its cup, grips your car... Gravity is a local something, and this local something is space contraction. While gravity can be described mathematically by the Newtonian force equations or by the abstractions of the general relativity equations, the actual mechanism of gravity is the inhomogeneous contraction of the space medium. [13].

The boundaries of the deSitter regions contain accumulations of mass - gravitational mass. And gravitational mass acts as the sink for the space medium. The regions that make up the boundaries are, therefore, dynamic. By way of some on-going process inherent in matter - a process involving absorption, assimilation, and dissipation- those regions actively contract the space medium.

A dual dynamic medium. The universe that is being modeled here has regions of space expansion and regions of space contraction; and it complies with the strong cosmological principle (more specifically, the perfect cosmological principle).

The essential point is that we now have a dual-dynamic space medium in a universe with large-scale homogeneity. And what happens when there are two opposing dynamic effects in a homogeneous environment?... Consider the evidence that we readily find in Nature: A fluid under the influence of both heating and cooling produces thermal convection cells; the expansion tendency of gas pressure versus the contraction tendency of surface tension leads to the formation of soap bubbles; there are freeze-thaw cycles that produce the polygonal terrain of the Northern Tundra; atomic forces versus macro stresses transforms amorphous ice into crystalline candled ice. All of these diverse examples have one thing in common - the formation of cells.

(a)

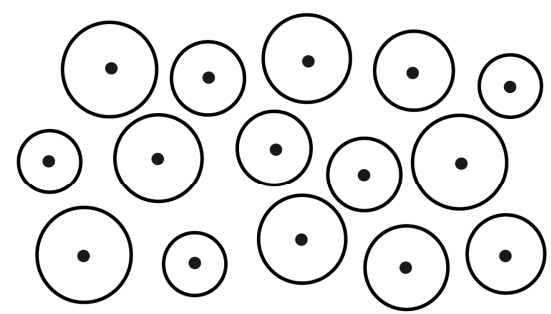

Expanding deSitter regions

(b)

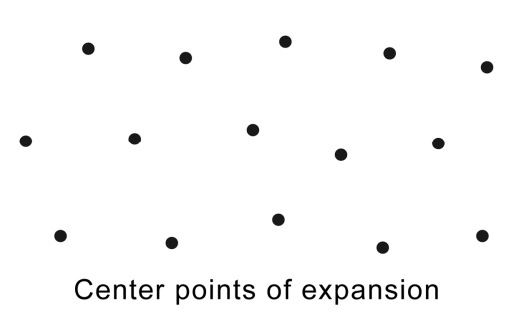

Fig. 1. Expanding deSitter regions, represented by circles in part (a), are randomly distributed in a plane. The individual center-points of expansion, shown in isolation in part (b), behave like repulsive particles. The Voronoi principle can be used to establish the final distribution pattern.

We should therefore expect that the dual dynamic processes of space-medium expansion and contraction will likewise involve a cell structure. We should expect the Universe to be partitioned into cells. They may be thought of as being somewhat like thermal convection cells except that the space medium does not circulate; rather, it flows in a one-way pattern. It flows radially, then "sinks" into, so called, Voronoi cell boundaries.

Let us consider in more detail the structuring of such cosmic cells.

\section{Voronoi Cells}

Imagine, for the sake of argument, a universe of solution $B$ 
cosmic bubbles and apply a simple geometric procedure called the theory of Voronoi cells.

Let us use a two-dimensional simplification before tackling the full three dimensions. Consider a planar universe with a random distribution of circular deSitter regions (Fig. 1a). In part (b) of Fig. 1 the circular boundaries have been removed leaving behind only the points to mark the geometric centers (GCs) of expansion. The points now represent the centers of deSitter's repulsive $\Lambda$-force, the centers of regional repulsion. The assumption is that all the GCs have the same capacity for repulsion and are free to move about.

The GCs, in an effort to distance themselves from their neighbors, can arrange into two possible ordered patterns: a Cartesian grid (Fig. 2a), and a symmetrical diagonal grid (Fig. 2b). In the Cartesian grid each GC is equidistant from its 4 closest neighbors, a distance designated as $d_{l}$. In the diagonal grid each GC is equidistant from 6 nearest neighbors, and this distance is labeled $d_{2}$ in the diagram.

The procedure for drawing a typical Voronoi cell is to choose a GC and connect it to each of the surrounding ones
(Fig. 2c \& d). On these radiating connectors draw perpendicular bisectors shown as dotted lines in (e) and (f).

Find the smallest convex region that is bounded by the bisector network surrounding the chosen GC. This region is known as the Voronoi cell. One of the distributions of GCs reveals a square cell and the other, a hexagonal Voronoi cell (Fig. 2g \& h).

By repeating the procedure we end up with two symmetrically tiled planes: one with squares the other with hexagons. Intuitively we know that the hexagonal pattern will be the natural outcome of the repulsive force. Here is the simple proof: Given that the distribution density of the GCs is constant and the system is in a state of equilibrium, each GC must occupy the same area regardless of the pattern. In other words the area of the square cell is equal to that of the hexagonal cell:

$$
\begin{aligned}
& \left(\text { Area }_{\text {HEXAGON }}\right)=\left(\text { Area }_{\text {SQUARE }}\right) \\
& 6 \times 1 / 2 d_{2} \times 1 / 2 d_{2} \times \tan 30^{\circ}=d_{1}^{2},
\end{aligned}
$$

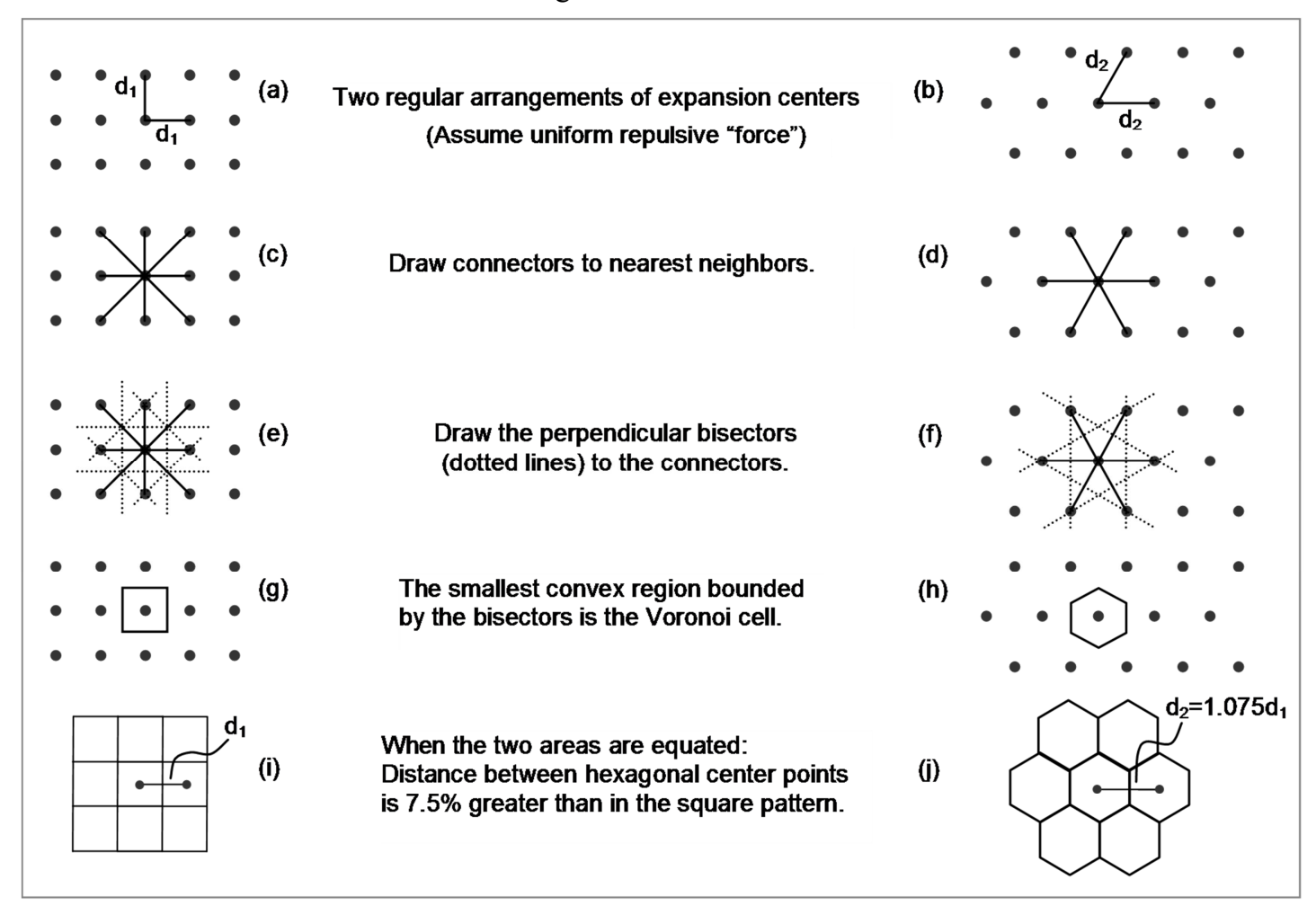

Fig. 2. Two possible regular arrangements, (a) and (b), of the center-points of expansion. It is assumed that each point is identically "repulsive" (that is, each deSitter region uses the same expansion parameter). The procedure for drawing the Voronoi cell around a point is to first mark connectors to its nearest neighbors, as in (c) and (d). Then draw the perpendicular bisectors (dotted lines) to the connectors, (e) and (f). The smallest convex region that is bounded by the bisector network surrounding the chosen center-point is the Voronoi cell, (g) and $(\mathrm{h})$. Since the density of the points in the plane is constant, the area of the Voronoi cells must be identical in both cases. By equating the two areas it is found that the distance between hexagonal center points is $7.5 \%$ greater than in the square pattern, (i) and (j).

and from this we can show that

$$
d_{2}=(2 / 3 \times \sqrt{ } 3)^{1 / 2} d_{1}=1.07456 d_{1} .
$$

Thus, the GCs, by "choosing" the hexagonal pattern, can distance themselves almost 7.5 percent farther from their neighbors than with the square pattern. In making this choice, the GCs fulfill their goal of maximizing distance which increases by a 1.075 factor without any change in the total area of its domain; only the shape of its domain is important. See Fig. 2 parts (i) and (j). 


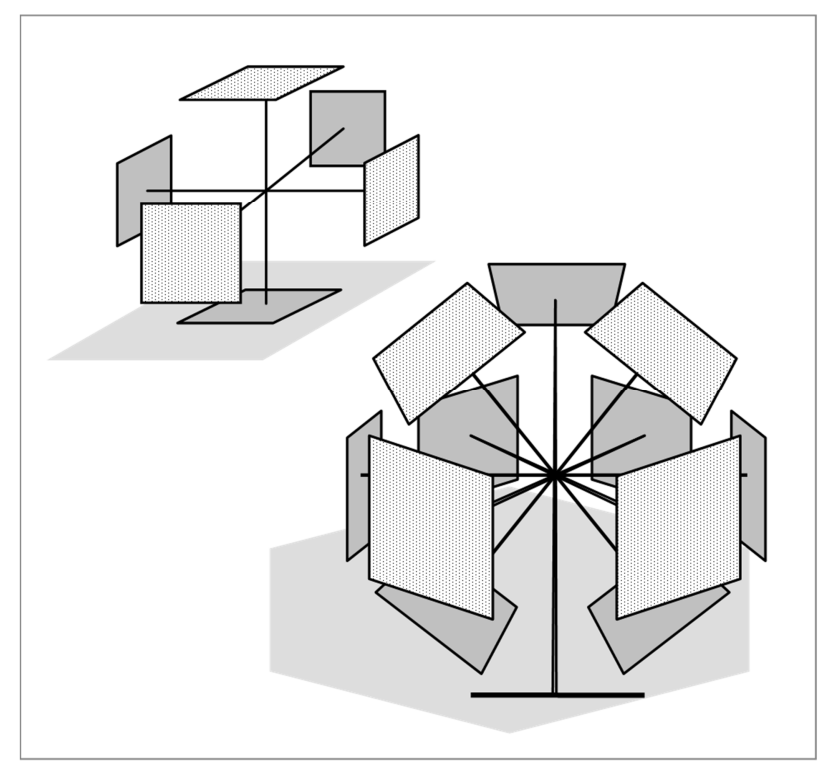

Fig. 3. Voronoi boundary planes, in exploded view, for the hexahedron (cube) and the dodecahedron.

A similar analysis applies to 3-dimensional space. Instead of expanding circles, we use expanding bubbles. Instead of drawing perpendicular bisector lines on the lines joining GCs we simply imagine perpendicular planes separating GCs. Each GC is then completely surrounded by perpendicular planes (Fig. 3). Again we will quantitatively compare patterns, this time between the cube, as a 3-dimensional version of a square, and the rhombic dodecahedron, as a 3-dimensional version of a hexagon. The density of the GCs in Euclidean background space is identical for both arrangements, and so the volume of the cube must be equal to the volume of the dodecahedron.

$$
\begin{gathered}
\left(\text { Volume }_{\text {DODECAHEDRON }}=\left(\text { Volume }_{\mathrm{CUBE}}\right)\right. \\
4 \times \sqrt{ } 2 \times\left(1 / 2 d_{\mathrm{D}}\right)^{3}=\left(d_{\mathrm{C}}\right)^{3}, \\
d_{\mathrm{D}}=(\sqrt{ } 2)^{1 / 3} \times d_{\mathrm{C}}=1.1224 d_{\mathrm{C}},
\end{gathered}
$$

where $d_{D}$ is the distance between adjacent dodecahedra GCs; $1 / 2 d_{D}$ is the inscribed-sphere radius of a dodecahedron; and $d_{C}$ is the distance between GCs of adjacent cubes.

Therefore, by using Voronoi cells ${ }^{2}$ with the rhombic dodecahedral shape instead of the hexahedral shape, the distance between geometric centers is greater by a substantial 12.25 percent.

What, then, is so important about the Voronoi cells sustained by deSitter's $\Lambda$-effect and Einstein's gravity-effect?... Well, it appears that our Universe is structured as Voronoi cells and the shape of the structures is predicted to be dodecahedral.

Now the Voronoi cell is a polyhedron. Astronomers have

2 Historical note: Georgii F. Voronoi (1868-1908) was a Russian mathematician who worked on number theory and multidimensional tilings. The concept of boundary formation has many diverse applications and not surprisingly has been 'rediscovered' many times. The cells could just as well be called Dirichlet domains and Wigner-Seitz cells. (Ian Stewart, Scientific American May 1998 p102-3) recently discovered that the large-scale distribution of matter in the universe resembles a network of such polyhedra. Most galactic clusters seem to be located on the boundaries of neighboring Voronoi cells. This pattern has been called the Voronoi cell model of the universe... -Ian Stewart [14].

And in the words of one of the astronomers in the forefront of the discovery: "In the Voronoi model, centers of voids are located randomly, and clusters are placed as far from void centers as possible.... During dynamical evolution matter flows away from the low-density regions and forms filaments and clusters of galaxies."[15] After more than 30 years of dedicated research, Jaan Einasto of Tartu Observatory in the year 2003 stated, "observational evidence suggests that rich superclusters and voids form a quasi-regular network of scale $\sim 100-130 \mathrm{~h}^{-1} \mathrm{Mpc}$;" and "voids between superclusters have mean diameters about $100 \mathrm{~h}^{-1} \mathrm{Mpc}$." It appears the "Cellular large-scale structure may be the end of the fractal structure of the Universe."[16] In other words, the observations suggest that there are no bigger structures than the Voronoi polyhedral cells.

And how big are these cells? It all depends on one's theory of the Cosmos. It depends on one's choice of equation for redshift-to-distance conversion and choice of expansion parameter; but here is a ballpark estimate:

In the 1980's it was discovered that clusters of galaxies are organized into giant bubbles measuring some 300 million lightyears in diameter... [And] preliminary research indicates that the bubbles do indeed represent the top level of structure. -Timothy Ferris [17].

In the introduction quote, Henning Genz, one of the leading experts on the properties of space, instructs us to examine how the deSitter repulsion will manifest itself. And we have found a most plausible solution; remarkably, a standard physics solution. On the largest structural scale, $\Lambda$ manifests itself as the interior void of a bubble-like cosmic cell. On the grandest scale of all, $\Lambda$ (in conjunction with contractile gravity) manifests itself as the Cellular Universe.

Space expansion (as a generic Lambda) acts as a repulsion force that strives to maximize the distance between centers of expansion. These geometric centers represent the centers of the voids from which space expands. And they act like centers of anti-gravity, from which precipitating matter is conveyed outwards. The Voronoi boundaries become the highly interactive interface between cosmic cells. As the space inside the cells expands, star clusters and galaxies and other matter become concentrated along the common Voronoi boundaries.

With the foregoing discussion of cell structuring it is extremely important to realize that the cellular universe based on DSSU theory should not be thought of as a universe in which cells are seeded and then grow to maturity, but rather as a universe in which cells are merely sustained. I do not want to give the impression that cells are popping up here and there in random fashion - that speculative scenario belongs to Andrei Linde and his chaotic inflation and self-reproducing universe models. The DSSU cells simply exist as timeless patterns and are maintained by perpetual steady-state processes. 
The cosmology theory known as the DSSU has long predicted that the Universe is a tessellation of Voronoi cells in the shape of dodecahedrons. In the year 2015, a revolutionary paper [18] was published in which the shape of the observed cosmic cells was verified to be dodecahedral.

\section{Cosmic Building Block}

The bubble interior would be a void, but the bubble wall would be the site of vigorous activity. -Jeremiah P. Ostriker [19].

The ideal Voronoi shape and the ideal cosmic cell is the rhombic dodecahedron (Fig. 4). This polyhedral shape is known as a closest-packing structure - meaning that multiple units can be packed tightly together with no gaps. It would be rather pleasing if the universe were an endless repetition of this ideal shape. But Nature, on her grandest structural scale, has an asymmetry and possibly random flaws as well. The rhombic dodecahedron is the result of a cubic closest-packed arrangement of GCs. There is, however, another arrangement of GCs that fulfills all the requirements of Voronoi modeling - the resulting cell shares all the basic geometric properties of having the same number of faces, edges, and nodes; even the same volume, surface area, and inscribed sphere. The arrangement is known as hexagonal closest-packed and the shape is termed the rhombic-trapezoidal dodecahedron. Nature has structured the cellular universe as a semi-regular packing, using at least two polyhedral shapes, resulting in asymmetries (presumably random) between the cubic and the hexagonal packing arrangements. Cosmic bubbles may take the shape of either the rhombic or the rhombic-trapezoidal dodecahedron.

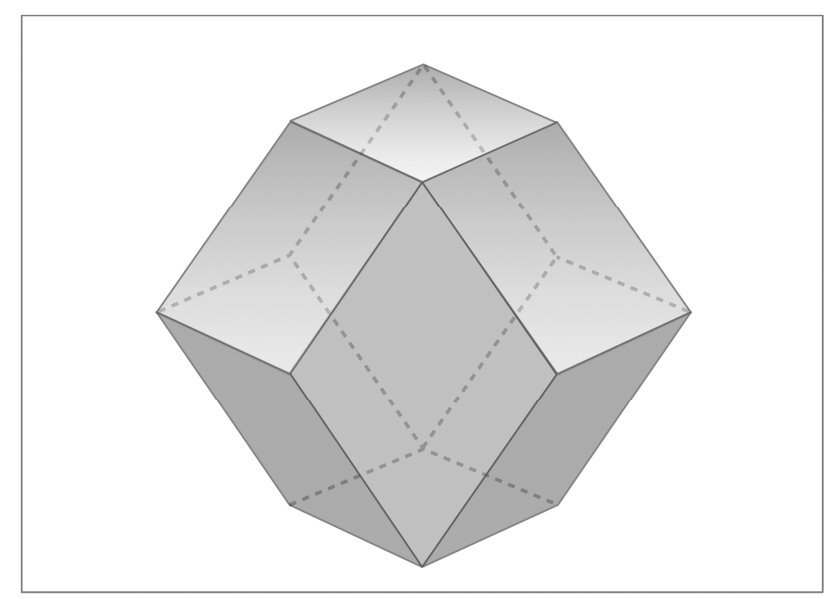

Fig. 4. Idealized rhombus-faced dodecahedral cosmic cell. This polyhedron shape is consistent with what is termed the cubic-closest packing of an assembly of deformable bubbles.

The shape of the cosmic cells is determined by the Voronoi principle; but the size of the cells is controlled by something else. The equilibrium between the rates of expansion and contraction determines the size. The equilibrium is the volumetric balance between the slow expansion of space within the cosmic bubble on the one hand, and the rapid contraction of space in the interface boundaries on the other. The two complementary rates are related in a self-adjusting system. In conventional terminology, we say that the generic Lambda effect is balanced by the gravitational effect; the interior expansion of the medium is balanced by boundary contraction of the medium. But the configuration is totally unconventional. Lambda is on the inside; gravity (and mass) is on the outside! Gravity prevents Lambda from enlarging the cosmic cells; while Lambda prevents gravity from collapsing the cells. Equilibrium is sustained and size is limited. This harmonious relationship means that if a cosmic bubble is hypothetically isolated, its size will retain stability (but it would, of course, lose its dodecahedral shape). ${ }^{3}$

3 In the maintenance of equilibrium between the expansion and contraction, a process of matter formation and annihilation also plays an essential role but not discussed in this article.
We can safely assume that the dynamic laws of Nature are universal, and, thus, conclude that all cosmic cells must tend towards some standard size. No doubt there is a constant adjustment of Voronoi boundaries - a territorial conflict in which any increase in the size of one bubble will be at the expense of its neighbors.

The building block of the DSSU is the dodecahedron: the closest-packed polyhedron. As an idealized unit the cubic closest-packed polyhedron has 12 identical rhombus faces, 24 edges, and 14 nodes. Although the faces are all identical, it cannot be classified as a regular polyhedron because some vertices connect three edges while others connect four; also, these two sets of vertices lie on separate circumscribing spheres. The hexagonal closest-packed polyhedron (not shown) consists of 6 rhombus and 6 trapezoid faces; consequently, it too is not a regular polyhedron. Both have numerous mirror and rotational symmetries and, as mentioned earlier, share all the basic geometric properties of having the same number of faces, edges, and nodes. Also their volume, surface area, and inscribed sphere and circumscribing spheres can be equated. 


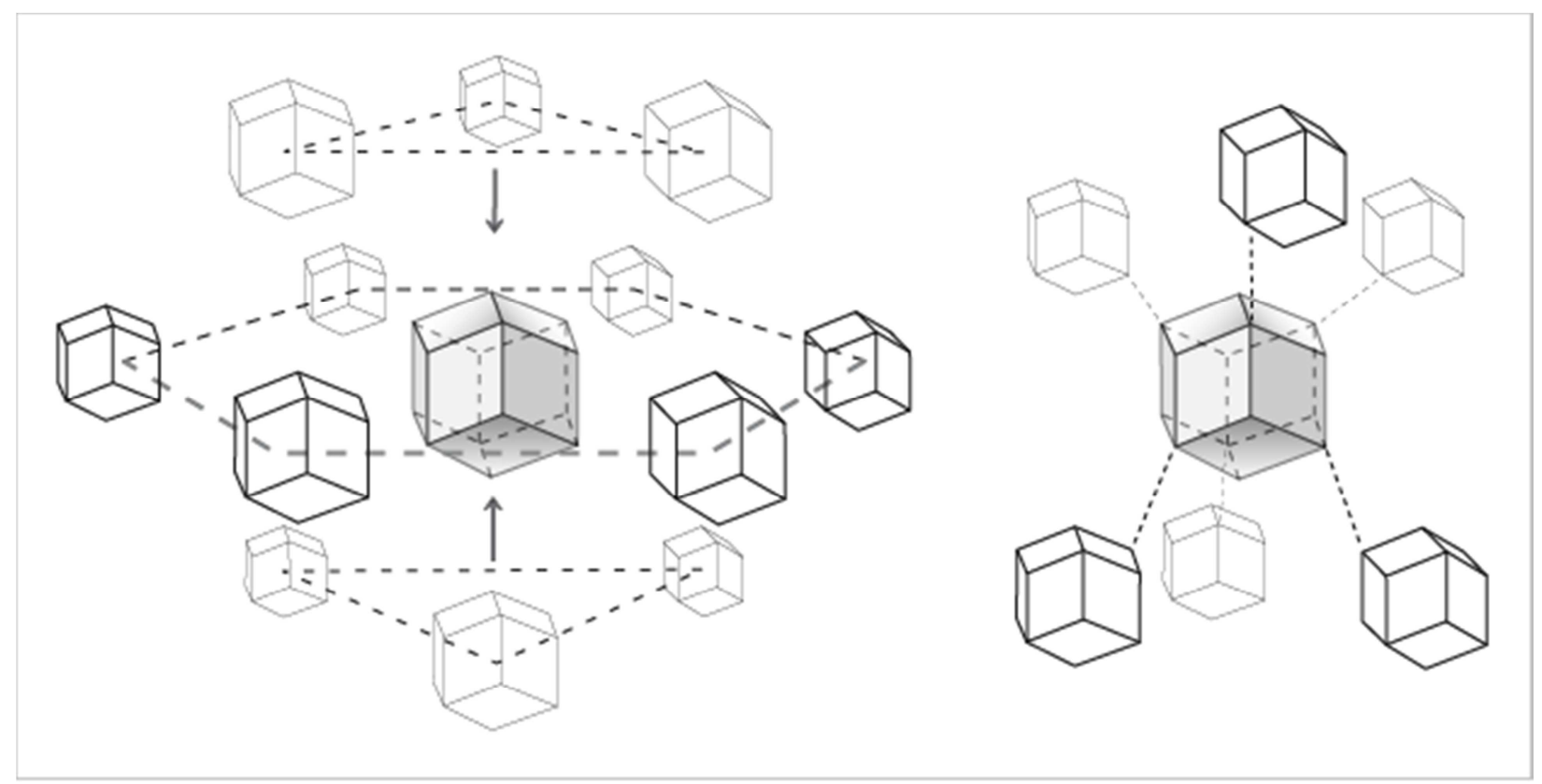

Fig. 5. Each cosmic cell is typically surrounded by eighteen similar cells. The exploded views show the twelve units that meet at the faces of the central cell (left) and the six units that only "touch" at respective major nodes (right).

The Dynamic Steady State Universe is an infinite array of such shapes. Within the array each cosmic cell is surrounded by 18 others. The exploded view (Fig. 5) gives some idea of how the units are actually "packed" together. Interestingly, although a typical dodecahedral cell has only 12 faces it actually interacts with 18 others (at least under idealized conditions). In a typical arrangement there are the obvious 12 units corresponding to the 12 faces, and then there are the less obvious 6 additional units that respectively connect to the 6 major nodes (of the central cell). An observer inside the central unit would be surrounded by an abundance of 14 nodes and 18 Voids! It is little wonder that our cosmic neighborhood appears rather chaotic.

Without this insight into the cellular geometry, it is unlikely that the true relationship between nodes and Voids - a relationship involving the dynamics and geometry of the DSSU's unified gravitation fields - would be decipherable.

\section{Large Scale Galaxy Structures}

Clusters tend to lie close to one another....[And] the voids are evidently an integral part of the process of clustering and superclustering. -Gregory and Thompson [20].

Any mass that is present in expanding space will, of course, move in the direction of expansion; and since expansion is radial, matter will comove with space in an explosion-like pattern. In effect, matter will freefall towards the outer boundaries and into the interface region, a region of aggregation. Each cosmic cells, in this manner, is accreting the material not only from its own interior but also that from the twelve (eighteen, more correctly) surrounding neighboring units.

At typical flat interface regions, the material from only two units aggregates (Fig. 6). One would expect to find vast sheets of star clusters and minor galaxies.

Concentration is even greater at a triple-interface boundary line. This is to be expected since space is expanding towards this boundary from three separate bubbles bringing with it comoving elliptical galaxies and the usual gas and dust clouds. It is here that one would expect to find a linear cluster, a galaxy cluster with a highly elongated longitudinal axis. A good example is the boundary in which our home galaxy is located. Gérard de Vaucouleurs in 1953, after years of observing and classifying galaxies, pointed out that most of the bright galaxies - from the local group neighbors to beyond the Virgo cluster- were confined to a narrow belt perpendicular to the Milky Way. In fact any galaxy cluster that astronomers term "filamentous" is the tracing of one or another of such boundary lines or edges.

And finally, the concentration of galaxies and debris is predicted to be greatest where four (or in some cases six) bubble universes meet at any of the dodecahedron's nodes. Here the material arrives from up to six sources. A clear and "nearby" example is the Virgo cluster; another is the core of the Coma cluster.

Sheets of galaxies, filamentous clusters, dense concentrations at nodes, and large voids are all features predicted by DSSU theory. The observational evidence is an overwhelming affirmation. Extensive redshift mappings of the Universe, such as those performed by Huchra et al. (1983), Giovanelli and Haynes (1986), De Lapparent et al. (1988), Broadhurst et al. (1990), Da Costa et al. (1994) and Vettolani et al. (1994) have discovered massive structures such as sheets, filaments, superclusters and voids, and have shown such structures to be repeating features of the observable universe. 


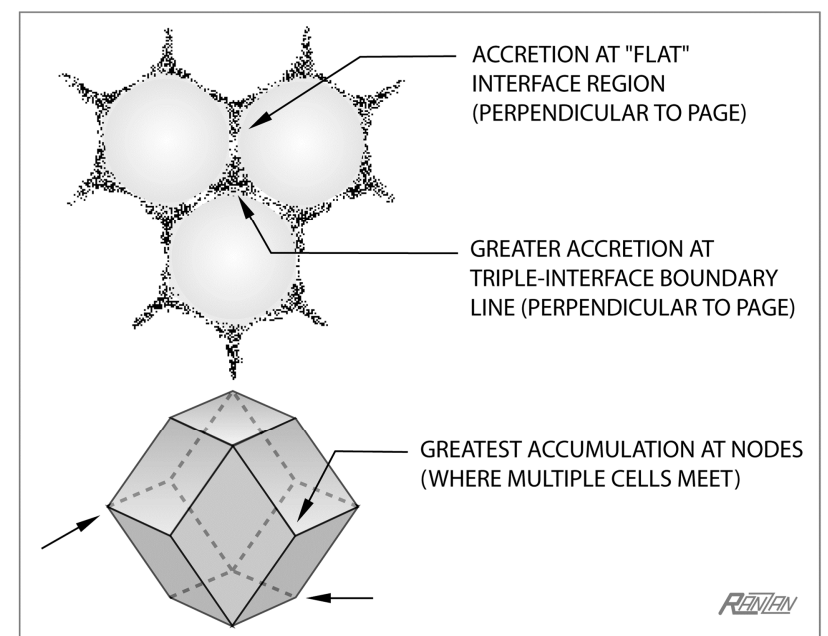

Fig. 6. Material, conveyed by comovement with expanding space, concentrates at flat interface regions, at triple-interface boundaries, and at nodes.

Margaret J. Geller, working at the Harvard-Smithsonian Center for Astrophysics has stated, "the pattern of galaxies in our three-dimensional slice of the universe suggested that sheets, or walls, containing thousands of galaxies mark the boundaries of vast dark regions nearly devoid of galaxies."[21].

Nodal structures. Each cosmic cell node consists of a symmetrical sub-structure. One of these structures has a tripod shape (Fig. 7 top); a shape derived from the boundary edges where four dodecahedra meet and share a common node. This region is where the galaxies from each of the adjacent four dodecahedra aggregate and form into a dense cluster. The other nodal structure is the quadrapod (Fig. 7 bottom) and is found at the nodes where six unit-universes meet. Many so called Abell galaxy clusters are the branches of the four-armed tripods or the eight-armed quadrapods.

The linking of such nodal clusters in various combinations forms the net or web-like pattern often described by astronomers (Fig. 8). By increasing the number of nodes and increasing the depth of view, an image emerges in which the structures link to reveal the skeletal Voronoi cells and a web-pattern of quadrilaterals -a ubiquitous pattern. Astronomer Einasto had found that the large scale organization of galaxies does have a net-like cellular structure with interconnected strings of galaxies surrounding empty regions. Laird Thompson and Stephen Gregory found that galaxies were never isolated but appeared to be joined to larger structures in chains or filaments with empty regions in between [22].

There can be little doubt that all extended superclusters represent the arrangement of a number of linked tripods and quadrapods having particularly favorable visibility.

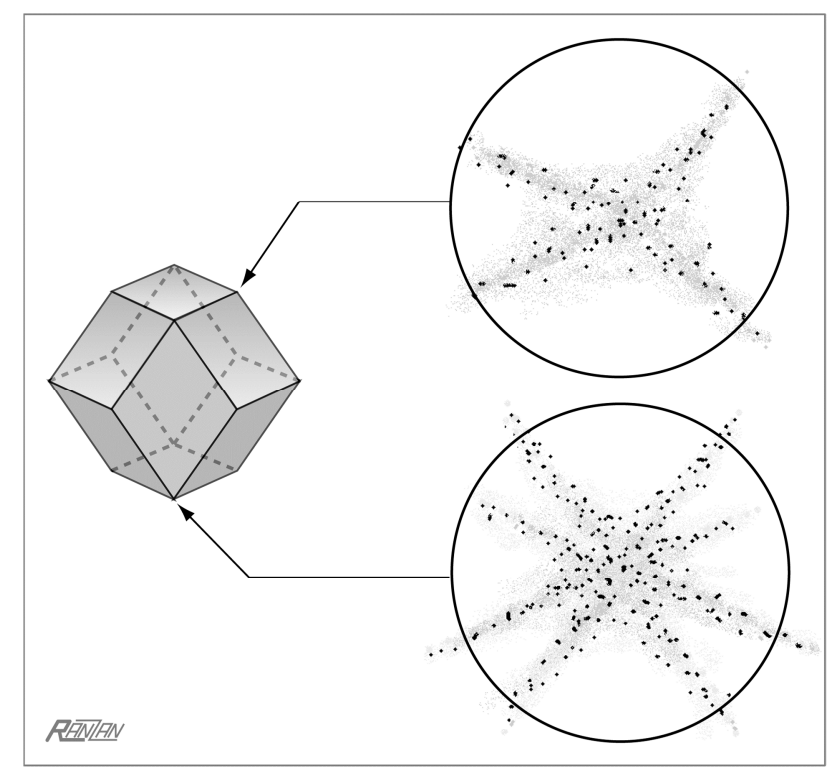

Fig. 7. Schematic of nodal structures. The 4-arm structure (top) occurs at nodes where four cosmic bubbles meet. The 8-arm structure (bottom) occurs at nodes where six bubbles meet. The dots represent galaxies and globular clusters.

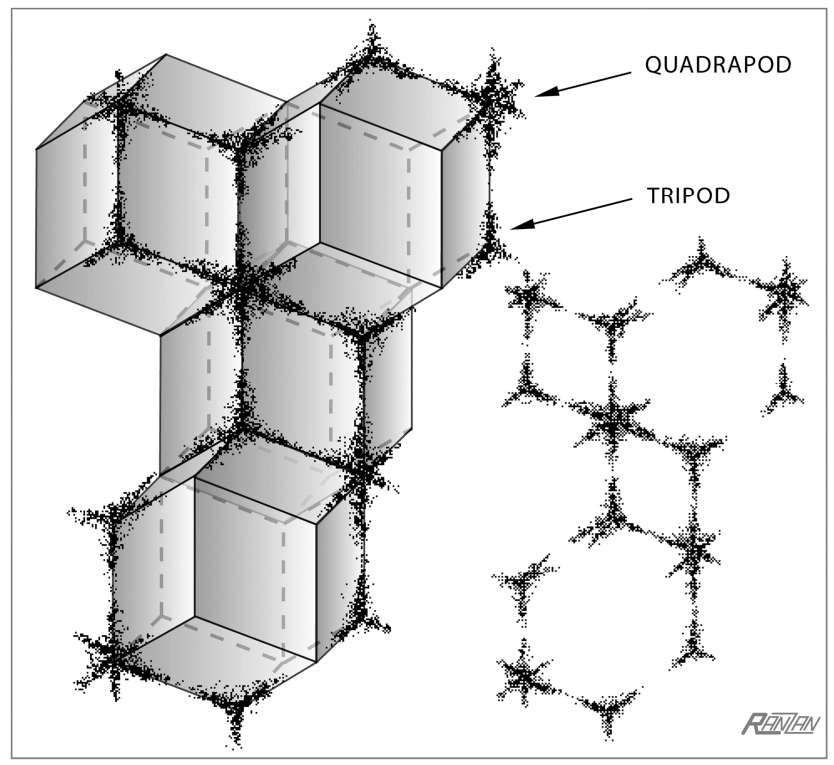

Fig. 8. The linking of multiple tripods and quadrapods presents a distinctive net-like pattern. Nodal structures are shown, in schematic, as they relate to a grouping of cosmic bubbles (left). On the right are the same structures without the dodecahedral framework. The nodes selected lie more or less in the same plane. 


\section{The Two Space-Medium Postulates of the DSSU}

At the heart of the theory of maintaining the cellular structure of the DSSU is a pair of space-medium postulates. At first glance it would appear that neither of the concepts embodied by these postulates is new. Expansion and contraction of space are ideas that have been around for a long time. What is new is the simultaneous expansion and contraction of space in spatially separated regions. Furthermore, the nature of the space medium is without precedence.

\subsection{The Space-Medium Defined}

In the new cosmology, space is defined as: (1) the essence medium of the universe; (2) a foam of subquantum fundamental fluctuators which are non-ponderable and therefore are devoid of mass and energy; and (3) having the property that it will expand when in its natural undisturbed state, or when subjected to cosmic tension; and (4) having the property that it will contract when in contact with matter, or when under pressure as within a gravitation region.

Space, as the essence medium of the universe, is a sea of subquantum fundamental fluctuators. These fluctuators are subquantum both in size (far below the Planck scale) and in quality - they do not represent energy as we understand energy to be. These essence-units of unimaginable minuteness serve as the precursors of all things.

The essence medium permeates the universe. It occupies the spatial interstices of matter and fills the "emptiness" of atoms. It conducts the electromagnetic force/energy.

\subsection{The Postulates}

Postulate \#1: Space, when subjected to a cosmic tension effect, expands; it expands in the sense that there is a quantitative increase in the number of fundamental fluctuators. In the absence of tension and pressure, space expands axiomatically.

The rate of expansion is extremely small. For the interior of a cosmic cells it has been calculated to be about 3.2 centimeters per kilometer of length every one million Earth years.

Historically space expansion has been called the deSitter effect. But it goes under several other names. Astronomers call it the Hubble expansion. Journalists describe it as a peculiar anti-gravitational effect. Big-bang proponents like to call it dark energy. Some astrophysicists take space expansion, speed it up, and call it inflation. Mathematicians consider space expansion as an abstraction and call it hyperbolic curvature of three- or four-dimensional space. The expansion of space is comparable to Einstein's positive-valued cosmological constant a "force" which he included in his calculations in an attempt to produce a balanced static universe (1917) based on his general relativity theory. Contemporary theorists consider space expansion as the manifestation of the vacuum energy.

In the big-bang genus of models, the expansion of space is responsible for the Hubble flow; and is, for the most part, universal and unconfined. But in the DSSU model, as we have seen, expansion is strictly confined within the bubble-like cosmic cells. The mechanism limiting the expansion is provided, in large part, by the second postulate.

Postulate \#2. Space contracts within mass bodies, mass objects, and all particles. Furthermore, it contracts within a space-contraction field surrounding gravitating mass bodies and particles. It contracts in the sense that there is a quantitative decrease in the number of fundamental fluctuators.

Although space contraction is a dynamic activity of the medium - and driven by the presence of matter - the action proceeds in steady state fashion. On the scale of the cosmic cells, the scale we are mainly interested in, space contraction is the steady state process that counters the steady state process of space expansion.

By this process the medium flowing from the Voids is consumed; that is, it is contracted out of existence by the gravitating bodies of the interface regions. A cell's equilibrium is thereby maintained; and the equilibrium state, in turn, determines the cell's size.

\subsection{Space and the Connection With Gravity}

Einstein's theory of gravitation is a geometric interpretation of gravity and mathematically describes the curvature distortion of some region of space. Gravity is the dynamic - the geometrodynamic - activity of geometrized space and geometrized time. The gravitational distortion of spacetime is a mathematical abstraction of something else — something that we recognize as quite real. Historically, the nature of the "something else" has never been made explicit. In fact, the nature of the distortion can never be made explicit within standard physics for the simple reason that Einstein's space is not absolute and is not supposed to have a substrate.

DSSU cosmology does make the explicit connection with reality. The model gives meaning to the gravitational distortion of space and defines it as the absorption and contraction of the space medium by matter. The contraction of space is the physical meaning behind the "distortion of spacetime" of general relativity theory. The "contraction of the space medium" is the mechanism of gravity.

\section{The New Dynamic Universe}

\subsection{The Search for Bubble Models}

It is worth repeating that the DSSU is not a bubble forming universe. The DSSU bubble structures are perpetually extant; they are not created. Bubble creation is an idea that is far too radical, wholly unrealistic, and quite unnecessary. I can't think of anything more radical than proposing different laws of physics for each bubble universe as does, for instance, the Self-Reproducing Inflationary Universe![23] However, in the 
desperate search to find alternate theories, the bubble formation idea seems attractive to some. One of the many versions of the Inflation-type models actually uses an exponential bubble-formation process and allows for arbitrary physical laws! Quoting from an article by Andrei Linde, a leading astrophysicist: "Recent versions of inflation theory assert that instead of being an expanding ball of fire the universe is a huge, growing fractal. It consists of many inflating balls that produce new balls, which in turn produce more balls, ad infinitum."'[24].

In another version, known as open inflation, being promoted by Professors Martin A. Bucher and David N. Spergel, "Bubble universes are self-contained universes that grow within a larger and otherwise empty 'multiverse'." Their model is by no means easy to explain. I fail to understand how they manage to make, in their words, "an entire hyperbolic universe (whose volume is infinite) to fit inside an expanding bubble (whose volume, though increasing without limit, is always finite)." No doubt they have an airtight mathematical explanation. Now, we may wonder, what about the close-packing of these bubbles. How do they fit together? How do they interact? Bucher and Spergel deal with this issue: "What if two bubbles collide? [Precisely!] Their meeting would unleash an explosion of cosmic proportions, destroying everything inside the bubbles near the point of impact.'[25] This self-destruction can't be good news. And yet this open inflation universe is supposed to be an expanding type of model.

Clearly, all bubble universes are not alike. Andrei Linde's universe has "self-generating" bubbles "sprouting" like weeds; Bucher and Spergel's inflationary universe has suicidal bubbles. And we haven't even mentioned Alan Guth's bubble universe; in his original version the bubbles were far too small, then in a later version they were far too big. As for the DSSU, it seems distinctly tame by comparison: no big bang, no bubble replication, no runaway inflation. In the DSSU, the inflation-like growth of the space medium is confined - in a steady-state manner-within staid and stable cosmic cells.

In the DSSU, inflation, which simply means space expansion, is quietly -in a steady-state-like mannercontained within an array of staid and stable cosmic cells.

Here is the all-important difference: Most of these postulated bubbles, and notably Andrei Linde's bubbles, are used to expand the universe as required by BB orthodoxy. The DSSU, in contrast, uses the bubbles as a quasi-static framework that holds the Universe in a steady state as required by the observational evidence.

\subsection{Gravitation Cells}

The emphasis in this article is on the physical structure of the Universe for which we assume a steady-state matter-formation process and a dual-dynamic substrate. The focus is on the visible cellular structure, the dodecahedra, as it is traced by matter and sustained by the dynamic activities of substrate expansion, contraction, and flow.

There is, however, another cellular configuration which has not been addressed but which should be briefly described. The
Universe is also divided into autonomous cosmic-scale gravitation cells. Although their boundaries are not visible, their centers are most obvious; their centers are the dominant galaxies within rich galaxy clusters. Each and every node of the dodecahedral structural cell marks the center of a cosmic gravitation cell. In the grand scheme of things, it is the gravitation cells, with their invisible boundaries, that sustain the Universe's visible cellular structure.

Furthermore, since the invisible boundaries of the gravity cells extend to the centers of the surrounding Voids, they may properly be referred to as unified gravitation cells (or what in a force-model would be termed fields). In other words, contractile gravity and Lambda become unified into autonomous gravitation regions.

\subsection{Cosmology's Lost Opportunity}

As the history of cosmology records, Einstein as well as deSitter did not pursue the line of reasoning that was used above and that led us to the cellular model, but instead treated the entire universe as one gigantic deSitter cell with a random sprinkling of gravitating mass. History also records that one of Einstein's greatest visions was finding a unifying link between the general relativity that governed the universe and the general relativity that governed the local worlds (and the laws that governed the quantum world).

In his 1917 cosmology paper, Einstein had tried to put together the broadest possible framework for how the universe might be constructed. He therefore envisioned two general kinds of influences that could affect the dynamics of the cosmos as a whole. Gravity bends space from without, causing collapse; Lambda unbends space from within, causing expansion... -Corey S. Powell [26].

Einstein never stopped searching for that unifying link between Lambda, the expander of space, and Gravity, the collapser of space. It seems the cellular configuration was never on the drawing board and was never explored. And the unifying connection was never found.

The search for the connection between Lambda and gravity was taken up by others and continued through the rest of the $20^{\text {th }}$ century. Often the focus was, and continues to be, on the conflict between what the conventional theory predicts and what the actual evidence demands. There is an on-going effort to try to explain the predicted large scale effects of a positive cosmological constant $(\Lambda)$ and the observed absence of such an effect. Various ideas are being examined.

One proposal is that some heretofore undiscovered symmetry in fundamental physics result in a cancellation of large effects, zeroing out the vacuum energy. -J. P. Ostriker [27].

The "symmetry" that theorists have been searching for is the symmetry manifest in the dynamic equilibrium state between $\Lambda$ and gravity - between space expansion and contraction. They know there is a cancellation and they know the expansion hypothesis they are using is wrong - the evidence says so- but they don't know how $\Lambda$ and gravity cancel each other. And it seems that for these many years since 1917 none has sought a solution in the pattern of the structure 
of the Universe.

In the beginning of the present century the unique dual dynamic nature of space was deciphered and presented as part of DSSU theory at the 2002 Munich Symposium. The link between Lambda the expander and Gravity the collapser had been found. The key that unlocks the mystery of the cancellation of the cosmological constant $(\Lambda)$ became available.

Figure 9 shows the harmonious link between the opposites of $\Lambda$ and gravity for one representative cell of our Universe. The DSSU consists of an infinite arrangement of such cells.

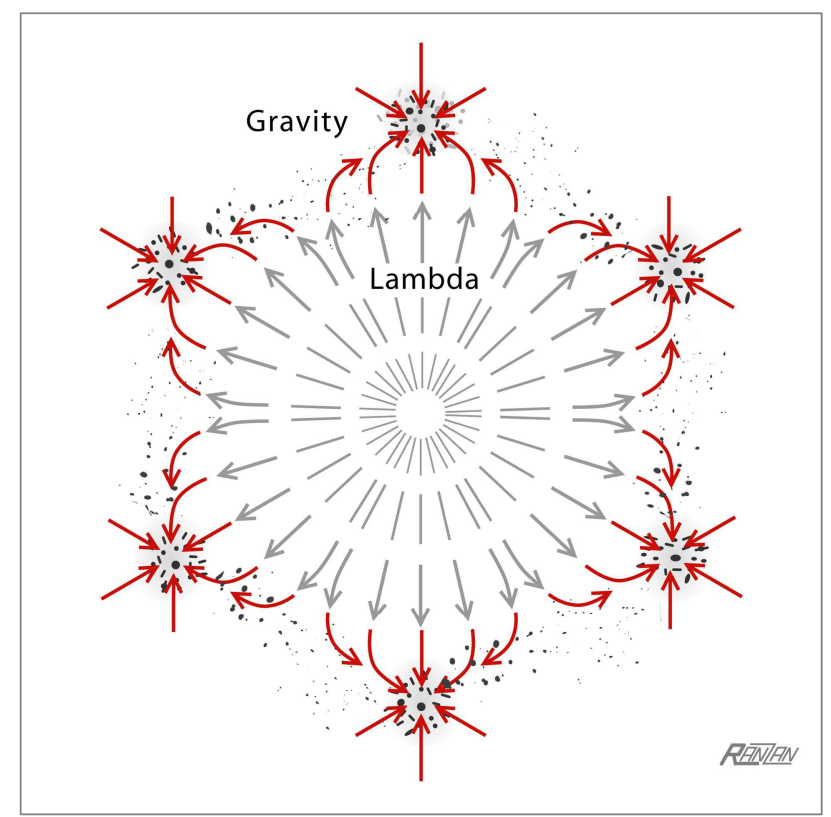

Fig. 9. Dual-dynamic cosmic cell. Schematic cross-section of a cosmic cell reveals the balance between the dynamic effects of Lambda and gravity. Space-medium expansion (gray arrows) is in equilibrium with space-medium contraction (red arrows).

\subsection{Classification and Summary}

The Dynamic Steady State Universe does not properly fit into the textbook classification system for types of universes.

Because the DSSU is a universe entirely permeated by a dynamic space medium, it is excluded from the static-universe class.

Because it has both expansion and contraction - and is quite unlike the historic models of Thomas Gold, Hermann Bondi, Fred Hoyle, and Jayant Narlikar - it fits rather poorly into the steady-state class.

And most definitely it is excluded from the dynamic expanding-universe class. No big bang, not even a little bang; and no steady-state whole-universe expansion.

Also note, the DSSU is not a fractal universe. A fractal universe is composed of a self-similar structure - one that repeats itself over and over on different scales. The DSSU is composed of a self-similar structure but only on one scale.

The DSSU is a unique model and properly fits only in the class of cellular universe (sub-class: non-expanding Euclidean) - a class not found in textbooks. Nevertheless, the DSSU includes all four aforementioned aspects. Its space is dynamic; its structural pattern tends to be static; its processes are continuous and perpetual and, therefore, steady state; and like a fractal model it has self-similar repeating units.

It should also be pointed out that the DSSU is not a multi-verse, not a multiplicity of universes. The DSSU is simple a multi-cellular universe.

What we have is a dynamic model within its cosmic cells; a steady state model in all its dynamic processes; and a static model on the largest scale. The defining feature is the expansion of space within a non-expanding universe. It is a universe in which the large structures such as galaxy clusters are not moving apart from each other.

There is one other classification category in which the DSSU is highly unusual. This has to do with the question of homogeneity and isotropy. The conventional wisdom has adopted the principle that the Universe is both homogeneous and isotropic. However, it can be shown that our universe is not isotropic.

On the scale of the typical polyhedral cell, with its near-empty Void surrounded by galaxy clusters, the DSSU is neither homogeneous nor isotropic. On the very largest scale, in which an extended array of bubbles is considered, the universe is very much like a vast crystal structure. And such a structure, by definition, always has preferential alignments, walls or planes that extend indefinitely. What this means is that the DSSU on such a scale may well be homogeneous but it cannot be isotropic! We might say that on the truly grandest scale this Universe is an awe-inspiring infinitely-facetted fluid crystal.

In summary, the DSSU is the cosmology theory based on a dynamic space-medium - a medium which continuously expands and contracts regionally and equally thereby sustaining a cosmic-scale cellular structure. It models the real world on the premise that all things are processes and that the space medium is the ultimate bedrock of Nature. Historically, it is the first true Steady State (SS) universe - SS nonexpanding, SS cellular, SS infinite, SS perpetual. For a detailed description of DSSU theory including its four main postulates, please see the article The Dynamic Steady State Universe [28] as well as articles [29] and [30].

\subsection{Closing Remarks}

Many leading scientists over the centuries, including Isaac Newton and Albert Einstein, believed that the universe is unchanging, neither contracting nor expanding. It now turns out that on this fundamentally important point they were right after all! With the recent discovery of the cell-sustaining processes of the Dynamic Steady State Universe it is possible to validate the view that the Universe does not expand - only the space medium expands. While the concept may sound paradoxical, it is simply that space expands within the Voronoi cells and simultaneously contracts at the Voronoi boundaries. The size of the cells does not change and neither does the greater universe. Whether we like it or not, the Universe is a sui generis steady state universe. But it is also a new dynamic universe and as such it represents an entirely new cosmology.

The New Cosmology represents a glorious simplification of 
the Universe since it can now be understood as an infinite Euclidean expanse arranged into non-Euclidean regions. The new picture reveals a non-dynamic framework of regions of dynamic space - of dual-dynamic space.

More than three centuries ago a new era in the study of biology began with the discovery that every individual animal and plant is composed of tiny living units, the cells.... A new era of cosmology begins with the recognition that the Universe is composed of dynamic-space units, the cosmic cells.

\section{References}

[1] Henning Genz, Nothingness, The Science of Empty Space (English translation edition, Perseus Books Publishing, 1999) p292.

[2] Halton Arp, Seeing Red: Redshifts, Cosmology and Academic Science (Apeiron, Montreal, 1998) Preface.

[3] Halton Arp, Seeing Red: Redshifts, Cosmology and Academic Science (Apeiron, Montreal, 1998).

[4] Hannes Alfvén, as in "The Big Bang Never Happened," Discover (June 1988).

[5] David Layzer, Constructing the Universe, Scientific American Library (W H Freeman \& Co., New York, 1984) p225.

[6] E. Hubble, "Effects of Red Shifts on the Distribution of Nebulae," Astrophysical Journal, Vol. 84, p. 517 (1936) (Posted at: http://adsabs.harvard.edu/abs/1936ApJ....84..517H)

[7] C. Ranzan, "Cosmic Redshift in the Nonexpanding Cellular Universe: Velocity-Differential Theory of Cosmic Redshift," American Journal of Astronomy \& Astrophysics, Vol. 2, No. 5, 2014, pp. 47-60. (Doi: 10.11648/j.ajaa.20140205.11)

[8] C. Ranzan, "DSSU Validated by Redshift Theory and Structural Evidence," Physics Essays, Vol. 28, No. 4, pp 455-473 (2015 Dec) (Doi: http://dx.doi.org/10.4006/0836-1398-28.4.455)

[9] Corey S. Powell, God in the Equation (The Free Press, New York, 2002) p83.

[10] Ibid., p88-89.

[11] C. Ranzan, "The Story of Gravity and Lambda -How the Theory of Heraclitus Solved the Dark Matter Mystery," Physics Essays Vol. 23, No. 1, p 75-87 (2010) (doi: http://dx.doi.org/10.4006/1.3293983) (Posted at: www.cellularuniverse.org/index.htm)

[12] Corey S. Powell, God in the Equation (The Free Press, New York, 2002) p84.

[13] R. T. Cahill, "Space and Gravitation," Magister Botanicus, Vol. 2, pp. 13-22, 2004.
[14] Ian Stewart, Scientific American (May 1998) p103.

[15] J. Einasto, "Large Scale Structure," Elsevier Preprint: arXiv:astro-ph/0011332v1 (Sept 2002) p14 \& 17.

[16] J. Einasto, "The Structure of the Universe on 100 Mpc Scales," in The Ninth Marcel Grossmann Meeting (2000 July). Proceedings ed. V. G. Gurzadyan, R. T. Jantzen, \& R. Ruffini, p291-300, 2002 (http://adsabs.harvard.edu/abs/2002nmgm.meet..291E) (Doi: 10.1142/9789812777386_0021)

[17] T. Ferris, The Whole Shebang (Simon \& Schuster, N. Y., 1997) p15.

[18] C. Ranzan, "DSSU Validated by Redshift Theory and Structural Evidence," Physics Essays, Vol. 28, No. 4, pp 455-473 (2015 Dec) (Doi: http://dx.doi.org/10.4006/0836-1398-28.4.455) (Posted at: www.cellularuniverse.org/index.htm)

[19] J. P. Ostriker and P. J. Steinhardt, "The Quintessential Universe," Scientific American Cosmos Edition (Dec 2002) p48.

[20] S. A. Gregory and L. A. Thompson, The Universe of Galaxies, Readings from Scientific American, Paul W. Hodge, editor (W. H. Freeman and Co., New York, 1984) p91 \& 94.

[21] M. J. Geller, “The Big Picture,” Natural History (2000 Feb).

[22] S. A. Gregory and L. A. Thompson, The Astrophysical Journal June 15, 1978 p784-799.

[23] Andrei Linde, "The Self-Reproducing Inflationary Universe," Scientific American Spring 1998 Special Edition Cosmos.

[24] Andrei Linde, "The Self-Reproducing Inflationary Universe," Scientific American Spring 1998 Special Edition Cosmos.

[25] Martin A. Bucher and David N. Spergel, "Inflation In a Low-Density Universe," Scientific American (Jan 1999) p62-69.

[26] Corey S. Powell, God in the Equation (The Free Press, New York, 2002) p195.

[27] J. P. Ostriker and P. J. Steinhardt, "The Quintessential Universe," Scientific American Cosmos Edition (Dec 2002) p44.

[28] C. Ranzan, “The Dynamic Steady State Universe," Physics Essays Vol. 27, No. 2, pp. 286-315 (2014 June) (doi: http://dx.doi.org/10.4006/0836-1398-27.2.286) (Posted at: www.cellularuniverse.org/index.htm)

[29] C. Ranzan, "The Processes of Gravitation -The Cause and Mechanism of Gravitation," Journal of Modern Physics and Applications, Vol. 2014 (2014) (Posted at: www.cellularuniverse.org/index.htm)

[30] C. Ranzan, "The Fundamental Process of Energy -A Qualitative Unification of Energy, Mass, and Gravity," Infinite Energy Issue \#113 Jan/Feb 2014 and Issue \#114 Mar/Apr 2014 (Posted at: www.cellularuniverse.org/index.htm) 